\title{
Hipertensão Arterial e Parâmetros Lipídicos, Glicídicos e de Adiposidade Associados em Adolescentes Escolares do Distrito Federal
}

\author{
Hypertension and Associated Lipid, Glucose, and Adiposity Parameters in School-Aged Adolescents in the \\ Federal District, Brazil
}

\author{
Letícia Rocha Lima, ${ }^{10}$ Aline Bassetto Okamura, ${ }^{2}$ Kênia Mara Baiocchi de Carvalho, ${ }^{2,3}$ Eliane Said Dutra, ${ }^{3}$ Vivian \\ Siqueira Santos Gonçalves ${ }^{2}$ \\ Departamento de Nutrição - Universidade de Brasília, ${ }^{1}$ Brasília, DF - Brasil \\ Programa de Pós-graduação em Saúde Coletiva - Universidade de Brasília, ${ }^{2}$ Brasília, DF - Brasil \\ Programa de Pós-graduação em Nutrição Humana - Universidade de Brasília, ${ }^{3}$ Brasília, DF - Brasil
}

\section{Resumo}

Fundamento: A prevalência de hipertensão arterial sistêmica (HAS) e de outros distúrbios metabólicos tem aumentado em indivíduos jovens. Entretanto, não há estudos representativos sobre esse assunto com a população do Distrito Federal (DF).

Objetivo: Estimar a prevalência de HAS e a sua associação com parâmetros lipídicos, glicídicos e de adiposidade em adolescentes do DF.

Métodos: Trata-se de um estudo observacional transversal com participantes do Estudo de Riscos Cardiovasculares em Adolescentes (ERICA). Foram avaliados pressão arterial, glicemia sanguínea, hemoglobina glicada, insulina, modelo de avaliação da homeostase da resistência à insulina (HOMA-IR), triglicerídeos, colesterol total, lipoproteína de alta densidade, lipoproteína de baixa densidade, índice de massa corporal (IMC) e perímetro da cintura, além de variáveis econômicas, demográficas e de maturação sexual. A análise de dados foi feita no software Stata e foi dividida nas seguintes etapas: análises descritiva, bruta e ajustada. Considerou-se $p<0,05$.

Resultados: Foram incluídos 1.200 adolescentes com média de idade de 14,8 anos. A prevalência de HAS foi de $8 \%$ (intervalo de confiança de 95\%: 6,3; 9,9). A maioria dos parâmetros se associou com a PA na análise bruta; na ajustada, os parâmetros glicídicos, lipídicos e de adiposidade mantiveram a associação, tendo IMC e HOMA-IR a maior magnitude na relação.

Conclusão: O estudo revelou elevada prevalência de HAS em adolescentes do DF, e os níveis pressóricos apresentaramse associados a outros marcadores de perfil lipídico, glicídico e de adiposidade, evidenciando a relevância da vigilância em saúde para o planejamento de ações efetivas para a reversão do quadro e prevenção de novos casos.

Palavras-chave: Hipertensão; Adolescente; Adiposidade; Glicemia; Lipídeos.

\begin{abstract}
Background: The prevalence of hypertension and other metabolic disorders has increased in young individuals. However, no representative studies have been conducted in the population of the Federal District, Brazil.

Objective: To estimate the prevalence of hypertension and its association with lipid, glucose, and adiposity markers in school-aged adolescents living in the Federal District.

Methods: This cross-sectional study included participants of the Study of Cardiovascular Risks in Adolescents (Portuguese acronym, ERICA). Blood pressure, blood glucose, glycated hemoglobin, insulin, homeostatic model assessment for insulin resistance (HOMA-IR), triglycerides, total cholesterol, high-density lipoprotein, low-density lipoprotein, body mass index (BMI), waist circumference, and economic, demographic, and sexual maturity variables were assessed. The data were analyzed in Stata, and the analysis was divided into different stages: descriptive, crude, and adjusted. Significant results were set at $p<0.05$
\end{abstract}

Results: In total, 1,200 adolescents were included, and their mean age was 14.8 years. The prevalence of hypertension was $8 \%$ (95\% confidence interval: 6.3; 9.9). Most parameters were associated with blood pressure in crude analysis. In adjusted analysis, glucose, lipid, and adiposity markers maintained the associations, and the highest magnitudes were those of BMI and HOMA-IR.

Correspondência: Letícia Rocha Lima •

Universidade de Brasília - Departamento de Nutrição - Faculdade de Ciências da Saúde. CEP 70910-900, Brasília, DF - Brasil

E-mail: Irochalima10@gmail.com

Artigo recebido em 25/11/2020, revisado em 08/03/2021, aceito em 12/05/2021

DOI: https://doi.org/10.36660/abc.20201240 
Conclusion: The study revealed a high prevalence of hypertension in adolescents living in the Federal District, and blood pressure levels were associated with other markers of lipid, glucose, and adiposity profile. The findings indicate the relevance of health surveillance for planning effective actions aimed at reversing this situation and preventing new cases.

Keywords: Hypertension; Adolescent; Adiposity; Blood Glucose; Lipids.

Full texts in English - http://www.arquivosonline.com.br

\section{Introdução}

As doenças crônicas não transmissíveis (DCNTs) tornaramse um problema de grande relevância para a saúde pública, protagonizando o cenário epidemiológico mundial junto às doenças cardiovasculares de ocorrência aguda. ${ }^{1}$ Entre as DCNTs mais prevalentes no mundo, destaca-se a hipertensão arterial sistêmica (HAS), uma condição clínica caracterizada por níveis elevados e sustentados da pressão arterial (PA), reconhecida por ser um importante fator de risco para doenças cardiovasculares, além de frequentemente estar associada a outros distúrbios metabólicos como obesidade, dislipidemias e intolerância à glicose. $^{2}$

A Organização Mundial de Saúde (OMS) estimou, em 2010, que cerca de 600 milhões de pessoas tinham diagnóstico de HAS, predizendo um crescimento global de $60 \%$ dos casos até 2025. ${ }^{3}$ No Brasil, dados da Pesquisa Nacional de Saúde (PNS), realizada em 2013, mostraram uma prevalência de 21,4\% da doença na população adulta. ${ }^{4}$ Paralelamente, tem sido observada uma mudança no perfil demográfico dos indivíduos com doenças crônicas, sendo cada vez mais comum a sua presença na infância e adolescência. ${ }^{5}$

As primeiras fases do curso da vida são importantes para o desenvolvimento humano, e alterações metabólicas precoces podem repercutir de maneira negativa na vida adulta, aumentando o risco para o desenvolvimento de doenças e comorbidades ao longo dos anos. ${ }^{6} \mathrm{O}$ Estudo de Riscos Cardiovasculares em Adolescentes (ERICA), realizado com escolares de todas as regiões brasileiras nos anos de 2013 e 2014, estimou a prevalência da doença em 9,6\% dos participantes. ${ }^{7}$ Uma revisão sistemática com metanálise, publicada em 2016, ${ }^{5}$ estimou a prevalência de HAS em $8 \%$ dos adolescentes brasileiros.

Tendo em vista esse cenário, a importância de monitorar o estado de saúde da população adolescente para auxiliar a tomada de decisão em ações de saúde e, ainda, a inexistência de estudos representativos sobre HAS e outros parâmetros metabólicos associados, conduzidos com a população adolescente do Distrito Federal (DF), o presente estudo se propõe a estimar a prevalência de HAS e investigar sua associação com parâmetros lipídicos, glicídicos e de adiposidade em adolescentes escolares do DF.

\section{Método}

\section{Delineamento e contexto do estudo}

Trata-se de um estudo observacional do tipo transversal realizado com participantes do ERICA entre 2013 e $2014 .^{8}$

\section{Critérios de elegibilidade}

Foram considerados elegíveis adolescentes de 12 a 17 anos, dos três últimos anos do Ensino Fundamental e do
Ensino Médio de escolas públicas e privadas, localizadas em áreas rurais e urbanas, sem qualquer deficiência, provisória ou definitiva, que nunca engravidaram e que aceitaram participar das coletas de sangue.

\section{Tamanho e seleção da amostra}

O ERICA teve representatividade em municípios de médio e grande porte, em nível nacional, regional e para cada capital incluída. Outros detalhes sobre a amostra nacional e a representatividade do estudo podem ser encontrados em Vasconcellos et al. ${ }^{9}$

No DF, a coleta de exames laboratoriais foi realizada em 33 escolas. Para a verificação da adequação do tamanho amostral para este estudo, considerou-se o total de 233.399 alunos no DF em 2009 nos últimos três anos do Ensino Fundamental e nos três anos do Ensino Médio, ${ }^{10}$ a prevalência de HAS na população adolescente escolar brasileira de 9\%, ${ }^{7}$ erro aceitável de $1,7 \%$ e nível de confiança de $95 \%$, totalizando número mínimo de 1.084 adolescentes.

Variáveis

\section{Pressão arterial}

As medidas de pressão arterial sistólica (PAS) e diastólica (PAD) foram consideradas variáveis de desfecho. Foi utilizado o aparelho oscilométrico automático, validado para adolescentes, Omron ${ }^{\circledR}$ 705-IT. ${ }^{11}$

Foram aferidas três medidas, com intervalo de 3 minutos entre cada uma; porém, foram consideradas apenas a média da segunda e da terceira medidas. ${ }^{8}$ Os adolescentes foram classificados de acordo com os valores de PAS e PAD em relação a estatura, sexo e idade, sendo $\geq$ percentil 95 classificados com HAS. ${ }^{12}$

\section{Coleta de exames laboratoriais}

A determinação dos parâmetros bioquímicos foi realizada a partir da coleta de amostra sanguínea por punção venosa, com jejum de 12 horas. ${ }^{13}$ A glicose sanguínea foi determinada a partir do método hexoquinase, e considerou-se valores $\geq 100 \mathrm{mg} / \mathrm{dL}$ elevados. ${ }^{14} \mathrm{~A}$ hemoglobina glicada (HbA1c) foi estimada por cromatografia de troca iônica, sendo consideradas elevadas as concentrações $\geq 5,8 \%$, valor correspondente ao percentil 90 para a população estudada. A insulina foi determinada pelo método de quimiluminescência e considerada elevada quando $\geq 15 \mathrm{mU} / \mathrm{L}^{15}$

O modelo de avaliação da homeostase da resistência à insulina (HOMA-IR) foi utilizado para caracterizar a resistência à insulina (RI) ${ }^{16}$ e calculado com da seguinte fórmula: insulina de jejum (mU/L) x (glicose de jejum 
$[\mathrm{mg} / \mathrm{dL}] \times 0,0555) / 22,5$. Foram considerados elevados os valores de HOMA-IR $\geq 2,80 .{ }^{17}$

O colesterol total (CT) e os triglicerídeos (TG) foram determinados pelo método de cinética enzimática, sendo consideradas elevadas as dosagens de CT $\geq 170 \mathrm{mg} / \mathrm{dL}$ e TG $\geq 90 \mathrm{mg} / \mathrm{dL} .{ }^{18}$ Avaliou-se, ainda, a lipoproteína de baixa densidade (LDL) e lipoproteína de alta densidade (HDL), sendo empregado o ensaio calorimétrico enzimático. Para $\mathrm{LDL}$, os valores considerados alterados foram $\geq 110 \mathrm{mg} / \mathrm{dL}$; para $\mathrm{HDL}, \leq 45 \mathrm{mg} / \mathrm{dL} .{ }^{18}$

\section{Parâmetros relacionados à adiposidade corporal}

Para aferição de peso e estatura, foram utilizados, respectivamente, balança eletrônica $\left(\right.$ Líder $\left.^{\circledR}\right)$, com capacidade de 200 kg e precisão de 50g, e estadiômetro portátil (Alturexata ${ }^{\circledR}$ ), com precisão de $1 \mathrm{~mm}$ e campo de uso de até $213 \mathrm{~cm}$. A estatura foi aferida em duplicata, permitindo a variação máxima de $0,5 \mathrm{~cm}$ entre as duas medidas. A média foi calculada automaticamente por sistema desenvolvido para uso em personal digital assistant (PDA). ${ }^{8}$

O índice de massa corporal (IMC) foi calculado a partir da divisão do peso (em quilogramas) pelo quadrado da altura (em metros). Foram utilizadas as referências da OMS ${ }^{19}$ para o cálculo dos escores-Z de IMC/idade, considerando o sexo. Foram considerados os seguintes pontos de corte: escore- $Z<-2$ (baixo peso); escore- $Z \geq-2$ e $<1$ (eutrofia); escore- $Z \geq 1$ e $<2$ (sobrepeso); escore- $Z \geq 2$ (obesidade).

Para aferição do perímetro da cintura (PC), foi utilizada fita antropométrica com resolução milimétrica e 1,5 $\mathrm{m}$ de comprimento $\left(\right.$ Sanny ${ }^{\circledR}$ ), adotando-se a técnica do ponto médio entre a crista ilíaca e a menor margem costal. A medida foi coletada em duplicata, e a média foi calculada. ${ }^{8}$ Os pontos de corte considerados foram os valores $\geq$ percentil 90 para a população estudada.

\section{Variáveis demográficas e econômicas}

As variáveis foram autorreferidas e assim categorizadas: sexo (feminino e masculino), idade ( $<15$ e $\geq 15$ anos) e cor da pele ou etnia (brancos, pardos, negros, indígenas, asiáticos e não referido). A localização (rural e urbana) e a rede de ensino (pública e privada) da escola foram identificadas, sendo essa última variável utilizada como proxy da classe econômica da família.

\section{Maturação sexual}

Os adolescentes foram classificados em diferentes estágios de maturação sexual, de acordo com o instrumento proposto por Tanner. ${ }^{20}$ Categorizou-se a partir da característica mais desenvolvida apontada, sendo os estágios 4 e 5 classificados como púberes e os demais, como não púberes.

\section{Análise dos dados}

Na etapa descritiva, calculou-se a prevalência e a distribuição das características de interesse na população estudada, bem como a prevalência de HAS em relação a essas características. Também foi realizada a comparação da prevalência de alterações em parâmetros bioquímicos e antropométricos entre os adolescentes com e sem HAS. Os resultados foram apresentados junto aos seus intervalos de confiança de 95\% (IC 95\%).

$\mathrm{Na}$ fase analítica, utilizou-se a técnica de regressão linear para investigar a associação entre PAS e PAD (variáveis dependentes) e marcadores laboratoriais e antropométricos (variáveis independentes). Essa fase foi dividida em duas etapas, análise bruta e análise ajustada, e foram utilizadas as seguintes variáveis para o ajuste: sexo, idade, estágio de maturação sexual, cor da pele ou etnia, obesidade e rede da escola. Quando a variável independente se referiu ao IMC ou ao PC, não houve ajuste pelo status de obesidade. Os resultados foram apresentados por meio do coeficiente $\beta$ da regressão com IC 95\%. A análise ajustada foi realizada somente quando a análise bruta apresentou $\mathrm{p}<0,20$, e considerou-se significativo $p<0,05$.

O desenho da amostra complexa e os respectivos pesos amostrais relacionados à população adolescente estudante do DF foram considerados. Foi utilizado o software Stata, versão 14.2 .

\section{Aspectos éticos}

O projeto foi aprovado pelo Comitê de Ética em Pesquisa com Seres Humanos da Faculdade de Medicina, Universidade de Brasília (CAAE Nº 05185212.2.2005.5540). Os participantes foram previamente informados acerca dos objetivos e procedimentos da pesquisa e foram avaliados somente após assinados os Termos de Assentimento pelo estudante e de Consentimento Livre e Esclarecido por seus pais ou responsáveis.

\section{Resultados}

Foram avaliados 1.200 adolescentes de 33 escolas públicas e privadas do Distrito Federal (DF). A média de idade foi de 14,8 anos, e a prevalência de HAS, de 8,0\% (IC 95\%: 6,3; $9,9)$, sendo identificada com maior frequência em estudantes do sexo masculino, com idade $\geq 15$ anos e que estudavam em escolas da zona rural.

A análise dos marcadores sanguíneos revelou a hiperglicemia como a alteração de menor prevalência. A alteração mais prevalente foi o baixo valor de HDL. Outras características estão descritas na Tabela 1.

Observou-se maior prevalência de hiperinsulinemia em adolescentes com HAS. Os parâmetros de adiposidade foram maiores em escolares com HAS, quando comparados aos sem HAS (Tabela 2).

A maior parte dos parâmetros analisados se associou à PAS e PAD na análise bruta. Na análise ajustada, parâmetros glicídicos, lipídicos e de adiposidade mantiveram-se associados, tendo o IMC e o HOMA-IR apresentado maior magnitude nessa relação (Tabela 3).

\section{Discussão}

Este é o primeiro estudo a investigar HAS nos adolescentes escolares do DF, e a prevalência estimada foi semelhante 
Tabela 1 - Perfil dos adolescentes escolares e prevalência de hipertensão arterial sistêmica. Estudo de Riscos Cardiovasculares em Adolescentes, Distrito Federal, Brasil, 2013-2014

\begin{tabular}{|c|c|c|c|c|}
\hline \multirow[b]{2}{*}{ Característica } & \multicolumn{2}{|c|}{ Amostra total } & \multicolumn{2}{|c|}{ Adolescentes com HAS } \\
\hline & $\%$ & IC $95 \%$ & $\%$ & IC 95\% \\
\hline \multicolumn{5}{|l|}{ Localização da escola } \\
\hline Área urbana & 97,0 & 81,$4 ; 99,5$ & 7,4 & 5,$9 ; 9,0$ \\
\hline Área rural & 3,0 & 0,$4 ; 18,5$ & 27,7 & 24,$6 ; 30,8$ \\
\hline \multicolumn{5}{|l|}{ Rede de ensino } \\
\hline Rede pública & 55,2 & 37,$5 ; 71,5$ & 8,3 & 5,$8 ; 11,7$ \\
\hline Rede privada & 44,8 & 28,$4 ; 62,4$ & 7,6 & 5,$7 ; 9,8$ \\
\hline \multicolumn{5}{|l|}{ Sexo } \\
\hline Feminino & 50,4 & - & 4,3 & 2,$8 ; 6,2$ \\
\hline Masculino & 49,6 & - & 11,8 & 9,$3 ; 14,7$ \\
\hline \multicolumn{5}{|l|}{ Idade } \\
\hline$<15$ anos & 47,6 & - & 5,4 & 3,$8 ; 7,6$ \\
\hline$\geq 15$ anos & 52,4 & - & 10,3 & 7,$7 ; 13,5$ \\
\hline \multicolumn{5}{|l|}{ Cor da pele/etnia } \\
\hline Brancos & 35,6 & 30,$1 ; 41,3$ & 8,4 & 5,$3 ; 13,1$ \\
\hline Pardos & 53,5 & 48,$4 ; 58,4$ & 7,9 & 6,$1 ; 10,1$ \\
\hline Negros & 6,0 & 4,$4 ; 8,2$ & 8,9 & 3,$9 ; 19,1$ \\
\hline Indígenas & 0,2 & 0,$0 ; 0,6$ & 16,7 & 1,$5 ; 71,5$ \\
\hline Asiáticos & 2,7 & 1,$7 ; 4,1$ & 3,5 & 0,$8 ; 13,6$ \\
\hline Não referido & 2,0 & 1,$3 ; 3,1$ & 2,8 & 0,$3 ; 18,7$ \\
\hline \multicolumn{5}{|c|}{ Estágio de maturação sexual* } \\
\hline Púbere & 81,9 & 78,$2 ; 85,0$ & 8,0 & 6,$3 ; 10,1$ \\
\hline Pré-púbere & 18,1 & 14,$9 ; 21,7$ & 7,7 & 3,$6 ; 15,5$ \\
\hline \multicolumn{5}{|l|}{ Glicose sanguínea $^{\dagger}$} \\
\hline$\geq 100 \mathrm{mg} / \mathrm{dL}$ & 1,5 & 0,$7 ; 3,2$ & 28,1 & 8,$4 ; 62,4$ \\
\hline \multicolumn{5}{|l|}{$\mathrm{HbA1c^{ \ddagger }}$} \\
\hline$\geq 5,8 \%(\geq p 90)$ & 13,6 & 10,$7 ; 17,1$ & 7,5 & 3,$6 ; 14,8$ \\
\hline \multicolumn{5}{|l|}{ Insulina§ } \\
\hline$\geq 15 \mathrm{mU} / \mathrm{L}$ & 11,3 & 8,$2 ; 15,5$ & 17,6 & 11,$6 ; 25,8$ \\
\hline \multicolumn{5}{|l|}{ HOMA-IR/I } \\
\hline$\geq 2,80$ & 18,2 & 13,$9 ; 23,5$ & 15,7 & 10,$7 ; 22,4$ \\
\hline \multicolumn{5}{|l|}{ Triglicerídeos" } \\
\hline$\geq 90 \mathrm{mg} / \mathrm{dL}$ & 30,5 & 27,$4 ; 33,8$ & 9,4 & 6,$3 ; 14,0$ \\
\hline \multicolumn{5}{|l|}{ Colesterol total" } \\
\hline$\geq 170 \mathrm{mg} / \mathrm{dL}$ & 30,6 & 27,$6 ; 33,7$ & 9,2 & 5,$9 ; 13,8$ \\
\hline \multicolumn{5}{|l|}{ LDL" } \\
\hline$\geq 110 \mathrm{mg} / \mathrm{dL}$ & 21,3 & 19,$0 ; 23,7$ & 7,2 & 4,$7 ; 10,8$ \\
\hline \multicolumn{5}{|l|}{ HDL" } \\
\hline$\leq 45 \mathrm{mg} / \mathrm{dL}$ & 41,8 & 38,$1 ; 45,4$ & 9,5 & 7,$2 ; 12,5$ \\
\hline \multicolumn{5}{|l|}{ IMC } \\
\hline Baixo peso e eutrofia & 77,0 & 73,$8 ; 79,9$ & 4,2 & 2,$9 ; 6,0$ \\
\hline Sobrepeso & 14,7 & 12,$3 ; 17,4$ & 16,0 & 10,$8 ; 23,0$ \\
\hline Obesidade & 8,3 & 6,$3 ; 10,6$ & 28,6 & 18,$2 ; 41,9$ \\
\hline \multicolumn{5}{|l|}{ Perímetro da cintura** } \\
\hline Não elevado (< p90) & 88,4 & 84,$8 ; 91,1$ & 6,2 & 4,$8 ; 7,8$ \\
\hline Elevado $(\geq \mathrm{p} 90)$ & 11,6 & 8,$8 ; 15,1$ & 21,6 & 14,$2 ; 31,3$ \\
\hline
\end{tabular}

HAS: hipertensão arterial sistêmica; IC 95\%: intervalo de confiança de 95\%; HbA1c: hemoglobina glicada; HOMA-IR: modelo de avaliação da homeostase da resistência à insulina; LDL: lipoproteína de baixa densidade; HDL: lipoproteína de alta densidade; IMC: índice de massa corporal. *Tanner, 1962; †SBD, 2019; ¥valores $\geq 5,8 \%$ (correspondente ao percentil 90 para a população estudada); \$SBC, 2005; "Chissini et al., 2019; "ISBC, 2017; \#OMS, 2007; **alores $\geq 80,8 \mathrm{~cm}$ (feminino) ou $\geq 86,3 \mathrm{~cm}$ (masculino). 
Tabela 2 - Prevalência de alterações bioquímicas e do estado nutricional em adolescentes com e sem hipertensão arterial sistêmica Estudo de Riscos Cardiovasculares em Adolescentes, Distrito Federal, Brasil, 2013-2014

\begin{tabular}{|c|c|c|c|c|}
\hline \multirow[b]{2}{*}{ Parâmetro avaliado } & \multicolumn{2}{|c|}{$\begin{array}{l}\text { Adolescentes } \\
\text { com HAS }\end{array}$} & \multicolumn{2}{|c|}{$\begin{array}{l}\text { Adolescentes } \\
\text { sem HAS }\end{array}$} \\
\hline & $\%$ & IC 95\% & $\%$ & IC $95 \%$ \\
\hline \multicolumn{5}{|l|}{ Glicose sanguínea* } \\
\hline$\geq 100 \mathrm{mg} / \mathrm{dL}$ & 5,4 & 1,$3 ; 18,8$ & 1,1 & 0,$5 ; 2,5$ \\
\hline \multicolumn{5}{|l|}{$\mathrm{HbA1} \mathrm{c}^{\dagger}$} \\
\hline$\geq 5,8 \%(\geq p 90)$ & 12,7 & 6,$0 ; 25,1$ & 13,7 & 10,$9 ; 17,1$ \\
\hline \multicolumn{5}{|l|}{ Insulina $a^{\ddagger}$} \\
\hline$\geq 15 \mathrm{mU} / \mathrm{L}$ & 25,4 & 13,$8 ; 41,8$ & 10,2 & 7,$5 ; 13,6$ \\
\hline \multicolumn{5}{|l|}{ HOMA-IR ${ }^{\S}$} \\
\hline$\geq 2,80$ & 35,9 & 23,$8 ; 50,1$ & 16,7 & 12,$7 ; 21,6$ \\
\hline \multicolumn{5}{|l|}{ Triglicerídeos ${ }^{\prime \prime}$} \\
\hline$\geq 90 \mathrm{mg} / \mathrm{dL}$ & 36,3 & 26,$3 ; 47,6$ & 30,0 & 27,$0 ; 33,2$ \\
\hline \multicolumn{5}{|l|}{ Colesterol total" } \\
\hline$\geq 170 \mathrm{mg} / \mathrm{dL}$ & 35,1 & 23,$8 ; 48,4$ & 30,2 & 27,$1 ; 33,4$ \\
\hline \multicolumn{5}{|l|}{ LDL" } \\
\hline$\geq 110 \mathrm{mg} / \mathrm{dL}$ & 19,2 & 12,$2 ; 28,9$ & 21,4 & 19,$2 ; 23,9$ \\
\hline \multicolumn{5}{|l|}{$\mathrm{HDL}^{\prime \prime}$} \\
\hline$\leq 45 \mathrm{mg} / \mathrm{dL}$ & 50,0 & 36,$3 ; 63,8$ & 41,0 & 37,$6 ; 44,5$ \\
\hline \multicolumn{5}{|l|}{ IMC } \\
\hline Baixo peso e eutrofia & 40,7 & 29,$1 ; 53,4$ & 80,1 & 77,$0 ; 82,9$ \\
\hline Sobrepeso & 29,6 & 20,$3 ; 41,0$ & 13,4 & 11,$0 ; 16,1$ \\
\hline Obesidade & 29,6 & 18,$5 ; 43,8$ & 6,4 & 4,$7 ; 8,5$ \\
\hline \multicolumn{5}{|l|}{ Perímetro da cintura ${ }^{\#}$} \\
\hline Elevado ( $\geq$ p90) & 31,5 & 20,$0 ; 45,8$ & 9,9 & 7,$5 ; 12,8$ \\
\hline
\end{tabular}

HAS: hipertensão arterial sistêmica; IC 95\%: intervalo de confiança de 95\%; HbA1c: hemoglobina glicada; HOMA-IR: modelo de avaliação da homeostase da resistência à insulina; LDL: lipoproteína de baixa densidade; HDL: lipoproteína de alta densidade; IMC: índice de massa corporal. "SBD, 2019; 'tvalores $\geq 5,8 \%$ (correspondente ao percentil 90 para a população estudada); ${ }^{\ddagger} S B C$, 2005; ${ }^{\circledR}$ Chissini et al., 2019; "SBC, 2017; "OMS, 2007; "Valores $\geq 80,8 \mathrm{~cm}$ para sexo feminino ou $\geq 86,3 \mathrm{~cm}$ para sexo masculino (correspondente ao percentil 90 para a população estudada).

à encontrada para as regiões Centro-oeste $(8,7, \mathrm{IC} 95 \%$ : $7,9 ; 9,6)$, Norte $(8,4$, IC 95\%: 7,7; 9,2), Nordeste $(8,4$, IC 95\%: 7,$6 ; 9,2)$, Sudeste $(9,8$, IC $95 \%: 8,8 ; 11,0)$ e para a amostra nacional do ERICA $(9,6$, IC 95\%: 9,0; 10,3). Foi menor somente que a prevalência estimada para a região Sul $\left(12,5\right.$, IC 95\%: 11,0; 14,2). ${ }^{7}$ Foi verificada, ainda, elevada prevalência de alterações nos demais parâmetros bioquímicos e de adiposidade investigados, e as associações encontradas podem potencializar o risco cardiovascular nessa população.

De maneira semelhante à amostra nacional do ERICA, ${ }^{7}$ apesar de a maioria dos adolescentes estudarem na zona urbana, a HAS estava mais presente em escolas da zona rural. Uma hipótese explicativa relaciona-se ao fato de os ambientes rurais muitas vezes terem acesso limitado a serviços de saúde, dificultando o diagnóstico e tratamento de doenças crônicas como a HAS. ${ }^{21}$

A avaliação de parâmetros relacionados ao metabolismo glicídico dos adolescentes mostrou que alterações na glicemia em jejum foram menos prevalentes que nos demais parâmetros. No entanto, a avaliação isolada da glicemia é insuficiente para descartar alterações metabólicas, visto que, no início do quadro de RI, pode ocorrer a manutenção da glicemia dentro dos níveis de normalidade como consequência de uma possível hiperinsulinemia. ${ }^{14} \mathrm{~A}$ alteração na $\mathrm{HbA1c}$, que foi mais prevalente nesses estudantes, pode ser um melhor marcador na avaliação do controle glicêmico, por refletir mudanças na glicemia a longo prazo. ${ }^{22}$

Níveis elevados de glicose sanguínea favorecem a HAS por meio do aumento de débito cardíaco causado pela hiperosmolaridade induzida pelo quadro de hiperglicemia. ${ }^{23}$ O excesso de glicose sanguínea também pode acarretar a geração excessiva de espécies reativas de oxigênio (EROs), o que colabora com a disfunção endotelial. ${ }^{24}$ Quando prolongada, a hiperglicemia pode contribuir, ainda, para a geração de produtos finais de glicação avançada, que pioram o estresse oxidativo por ativar uma cascata pró-inflamatória, aumentando a expressão de EROs e contribuindo para inibição ou redução da produção de óxido nítrico, o que leva a resistência vascular periférica por vasoconstrição. ${ }^{24,25}$ 


\begin{tabular}{|c|c|c|c|c|}
\hline \multirow{2}{*}{ Parâmetro avaliado } & \multicolumn{2}{|c|}{ Análise bruta } & \multicolumn{2}{|c|}{ Análise ajustada* } \\
\hline & Coeficiente $\beta$ & IC $95 \%$ & Coeficiente $\beta$ & IC $95 \%$ \\
\hline \multicolumn{5}{|l|}{ PAS } \\
\hline Glicose (mg/dL) & $0,26^{\S}$ & 0,$13 ; 0,39$ & $0,16^{\ddagger}$ & 0,$04 ; 0,27$ \\
\hline HbA1c (\%) & $1,77^{\dagger}$ & $-0,84 ; 4,39$ & 0,68 & $-1,41 ; 2,78$ \\
\hline Insulina (mU/L) & $0,24^{\ddagger}$ & 0,$03 ; 0,45$ & $0,23^{\ddagger}$ & 0,$06 ; 0,40$ \\
\hline HOMA-IR & $1,19^{\ddagger}$ & 0,$17 ; 2,21$ & $1,07^{\ddagger}$ & 0,$25 ; 1,88$ \\
\hline TG (mg/dL) & $0,04^{\S}$ & 0,$02 ; 0,06$ & $0,03^{\ddagger}$ & 0,$01 ; 0,04$ \\
\hline CT (mg/dL) & $-0,001$ & $-0,03 ; 0,02$ & & \\
\hline LDL (mg/dL) & 0,008 & $-0,02 ; 0,03$ & & \\
\hline $\mathrm{HDL}(\mathrm{mg} / \mathrm{dL})$ & $-0,18^{\S}$ & $-0,27 ;-0,09$ & $-0,06^{\ddagger}$ & $-0,12 ;-0,002$ \\
\hline IMC $\left(\mathrm{kg} / \mathrm{m}^{2}\right)$ & $1,49^{\S}$ & 1,$28 ; 1,71$ & $1,41^{\S}$ & 1,$20 ; 1,63$ \\
\hline $\mathrm{PC}(\mathrm{cm})$ & $0,68^{\S}$ & 0,$58 ; 0,79$ & $0,58^{\S}$ & 0,$48 ; 0,69$ \\
\hline \multicolumn{5}{|l|}{ PAD } \\
\hline Glicose (mg/dL) & $0,10^{\ddagger}$ & 0,$01 ; 0,19$ & $0,08^{\ddagger}$ & 0,$002 ; 0,17$ \\
\hline $\mathrm{HbA1c}(\%)$ & $1,09^{\dagger}$ & $-0,56 ; 2,76$ & 0,65 & $-0,77 ; 2,07$ \\
\hline Insulina (mU/L) & $0,13^{\dagger}$ & $-0,05 ; 0,31$ & 0,09 & $-0,05 ; 0,24$ \\
\hline HOMA-IR & $0,62^{\dagger}$ & $-0,26 ; 1,51$ & 0,45 & $-0,25 ; 1,16$ \\
\hline TG (mg/dL) & $0,02^{\ddagger}$ & 0,$01 ; 0,03$ & $0,01^{\ddagger}$ & 0,$005 ; 0,02$ \\
\hline CT (mg/dL) & $0,02^{\ddagger}$ & 0,$00 ; 0,03$ & $0,02^{\ddagger}$ & 0,$007 ; 0,03$ \\
\hline LDL (mg/dL) & $0,01^{\ddagger}$ & 0,$00 ; 0,03$ & $0,01^{\ddagger}$ & 0,$002 ; 0,03$ \\
\hline $\mathrm{HDL}$ (mg/dL) & $-0,01$ & $-0,06 ; 0,02$ & & \\
\hline IMC (kg/m²) & $0,57^{\S}$ & 0,$43 ; 0,72$ & $0,56^{\S}$ & 0,$40 ; 0,72$ \\
\hline PC (cm) & $0,27^{\S}$ & 0,$20 ; 0,33$ & $0,25^{\S}$ & 0,$18 ; 0,33$ \\
\hline
\end{tabular}

IC 95\%: intervalo de confiança de 95\%; PAS: pressão arterial sistólica; HbA1c: hemoglobina glicada; HOMA-IR: modelo de avaliação da homeostase da resistência à insulina; TG: triglicerídeos; CT: colesterol total; LDL: lipoproteína de baixa densidade; HDL: lipoproteína de alta densidade; IMC: indice de massa corporal; $P C$ : perímetro da cintura; $P A D$ : pressão arterial diastólica. ${ }^{\dagger} p<0,20 ;{ }^{\ddagger} p<0,05 ;{ }^{\S} p<0,001 ;{ }^{*} A$ análise foi ajustada para sexo, idade, estágio de maturação sexual, cor da pele ou etnia, rede de ensino e presença ou não de obesidade.

Além disso, verificou-se elevação nos níveis de insulina e HOMA-IR. Andrade et al. ${ }^{26}$ sugerem, no entanto, que a RI pode estar relacionada ao desenvolvimento na adolescência, envolvendo alterações hormonais e de composição corporal em indivíduos de estágios iniciais da puberdade, podendo ser revertida após o estirão de crescimento. No entanto, isso não explica os resultados encontrados, pois a maior parte da população estudada encontrava-se na fase final da puberdade. Assim como entre os adolescentes do DF, outros estudos identificaram maiores níveis de insulina e alterações no HOMA-IR em adolescentes com HAS em relação aos demais. ${ }^{27,28}$

Os adolescentes com HAS também apresentaram maior IMC e PC, situação bem estabelecida em outros estudos. ${ }^{29,30} \mathrm{~A}$ adiposidade elevada contribui para o quadro de hipertensão, entre outros mecanismos, por favorecer o estresse oxidativo a partir da instalação de um estado pró-inflamatório com aumento na expressão de citocinas como interleucina 6 (IL-6) e fator de necrose tumoral $\alpha$. $^{31}$ A inflamação é um mediador importante tanto na instalação quanto na manutenção dos níveis pressóricos elevados pela lesão vascular e renal que pode causar. ${ }^{32}$ Além disso, indivíduos com obesidade podem apresentar hiperativação simpática, com aumento na produção de noradrenalina em nível renal e consequente aumento na reabsorção tubular de sódio. ${ }^{33} \mathrm{~A}$ hiperativação simpática pode ser ainda mais estimulada pela produção excessiva de leptina, comum em indivíduos com excesso de adiposidade. ${ }^{34} \mathrm{~A}$ presença de tecido adiposo não funcionante observada na obesidade pode alterar, ainda, o sistema renina angiotensina, aumentando os níveis circulantes de angiotensina II e aldosterona, causando alterações hemodinâmicas que também contribuem para a elevação da PA, ${ }^{35}$ justificando a associação observada no estudo.

A presença de marcadores de inflamação e disfunção endotelial também são perfis característicos das dislipidemias. ${ }^{36}$ Alterações de perfil lipídico e presença de citocinas inflamatórias como IL-6 se relacionam com o aumento da rigidez arterial e consequentemente com a pressão sanguínea, favorecendo a instalação do quadro hipertensivo 
e aumentando ainda o risco do desenvolvimento de doenças

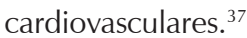

Estudos realizados em outras regiões do Brasil ${ }^{29,38} \mathrm{e}$ do mundo ${ }^{39,40}$ corroboram as prevalências e associações encontradas no DF, reforçando a frequência de fatores associados ao risco cardiovascular em idades cada vez mais precoces. ${ }^{41} \mathrm{O}$ estilo de vida não saudável da população adolescente brasileira, principalmente representado pela baixa qualidade da alimentação, ${ }^{42,43}$ sedentarismo e elevado tempo de tela, ${ }^{44}$ além do estresse emocional, ${ }^{45}$ potencializa os riscos apresentados.

A interpretação dos resultados do ERICA, no entanto, deve levar em consideração algumas de suas limitações. A prevalência de HAS pode ter sido superestimada pelo fato de a pressão ter sido aferida em um único dia. ${ }^{12} \mathrm{Em}$ estudos transversais de grande magnitude como o ERICA essa limitação é frequente, já que aumentar o número de visitas implica em maiores investimentos financeiros e logísticos. Vale ressaltar que a própria metodologia adotada no dia de coleta pode ter reduzido esse viés. ${ }^{8}$

Apesar dessa limitação, o ERICA contou com processos de monitoramento de qualidade ao longo de todo o período de coleta, além de grande cuidado metodológico adotado durante a análise estatística. Isso contribuiu para a robustez do estudo e a confiabilidade dos resultados encontrados.

\section{Referências}

1. World Health Organization. The top 10 Causes of Death [Internet]. Geneva: World Health Organization; c2018 [cited 2021 Jul 28]. Available from: https://www.who.int/news-room/fact-sheets/detail/the-top-10-causes-ofdeath.

2. Malachias MVB, Souza WKSB, Plavnik FL, Rodrigues CIS, Brandão AA, Neves MFT, et al. 7 Diretriz Brasileira de Hipertensão Arterial. Arq Bras Cardiol. 2016;107(3):1-103.

3. World Health Organization. Global Status Report on Noncommunicable Diseases. Geneva: World Health Organization; 2011

4. Theme Filha MM, Souza PR Jr, Damacena GN, Szwarcwald CL. Prevalence of Chronic Non-Communicable Diseases and Association With Self-Rated Health: National Health Survey, 2013. Rev Bras Epidemiol. 2015;18(Suppl 2):83-96. doi: 10.1590/1980-5497201500060008.

5. Gonçalves VS, Galvão TF, Andrade KR, Dutra ES, Bertolin MN, Carvalho KM, et al. Prevalence of Hypertension Among Adolescents: Systematic Review and Meta-Analysis. Rev Saude Publica. 2016;50:27. doi: 10.1590/S15188787.2016050006236

6. Lurbe E, Ingelfinger JR. Blood Pressure in Children and Adolescents: Current Insights. J Hypertens. 2016;34(2):176-83. doi: 10.1097/ HJH.0000000000000790.

7. Bloch KV, Klein CH, Szklo M, Kuschnir MC, Abreu GA, Barufaldi LA, et al. ERICA: Prevalences of Hypertension and Obesity in Brazilian adolescents. Rev Saude Publica. 2016;50(Suppl 1):9s. doi: 10.1590/ S01518-8787.2016050006685.

8. Bloch KV, Szklo M, Kuschnir MC, Abreu GA, Barufaldi LA, Klein CH, et al. The Study of Cardiovascular Risk in Adolescents--ERICA: Rationale, Design and Sample Characteristics of a National Survey Examining Cardiovascular Risk Factor Profile in Brazilian Adolescents. BMC Public Health. 2015;15:94. doi: 10.1186/s12889-015-1442-x.

\section{Conclusão}

A prevalência de HAS estimada em adolescentes escolares do DF foi de $8 \%$, associada a parâmetros metabólicos e de adiposidade. Esses achados evidenciam as interconexões metabólicas que podem estar presentes no quadro clínico hipertensivo. Nesse contexto, ações de promoção à saúde e prevenção de doenças são fundamentais para se evitar a situação evidenciada no DF, colaborando com a qualidade de vida da população e com menor impacto ao sistema de saúde vigente.

\section{Potencial conflito de interesse}

Não há conflito com o presente artigo

\section{Fontes de financiamento}

O presente estudo foi financiado pelo Departamento de Ciência e Tecnologia a Secretaria de Ciência e Tecnologia e Insumos Estratégicos do Ministério da Sáude (DECIT/SCTIE/ MS) e pelo Fundo Setorial de Sáude (CT-Saúde) do Ministério da Ciência, Tecnologia e Inovação (MCTI) (protocolos: FINEP-01090421 e CNPq-565037/2010-2).

\section{Vinculação acadêmica}

Não há vinculação deste estudo a programas de pósgraduação.
9. Vasconcellos MT, Silva PL, Szklo M, Kuschnir MC, Klein CH, Abreu GA, et al Sampling Design for the Study of Cardiovascular Risks in Adolescents (ERICA). Cad Saude Publica. 2015;31(5):921-30. doi: 10.1590/0102-311X00043214.

10. Instituto Nacional de Estudos e Pesquisas Educacionais. Relatório Técnico: Resultado do Censo da Educação Básica 2009. Brasília: Ministério da Educação; 2010. p. 1-19.

11. Stergiou GS, Yiannes NG, Rarra VC. Validation of the Omron 705 IT Oscillometric Device for Home Blood Pressure Measurement in Children and Adolescents: the Arsakion School Study. Blood Press Monit. 2006;11(4):22934. doi: 10.1097/01.mbp.0000209074.38331.16.

12. National High Blood Pressure Education Program Working Group on High Blood Pressure in Children and Adolescents. The Fourth Report on the Diagnosis, Evaluation, and Treatment of High Blood Pressure in Children and Adolescents. Pediatrics. 2004;114(2 Suppl):555-76.

13. Cureau FV, Bloch KV, Henz A, Schaan CW, Klein CH, Oliveira CL, et al. Challenges for Conducting Blood Collection and Biochemical Analysis in a Large Multicenter School-Based Study With Adolescents: Lessons From ERICA in Brazil. Cad Saude Publica. 2017;33(4):e00122816. doi: 10.1590/0102$311 \times 00122816$

14. Forti AC, Pires AC, Pittito BA, Gerchman F, Oliveira JEP, Zajdenverg L, et al. Diretrizes da Sociedade Brasileira de Diabetes 2019-2020. Soc Bras. Diab. Arq. Bras. Cardiol. 2019;53:1689-99.

15. Giuliano ICB, Caramelli B, Pellanda L, Duncan B, Mattos S, Fonseca FH, et al. I Diretriz de Prevenção da Aterosclerose na Infância e na Adolescência. Arq Bras Cardiol. 2005;85(6):1-36. doi: 10.1590/S0066-782X2005002500001.

16. Matthews DR, Hosker JP, Rudenski AS, Naylor BA, Treacher DF, Turner RC. Homeostasis Model Assessment: Insulin Resistance and Beta-Cell Function From Fasting Plasma Glucose and Insulin Concentrations in Man. Diabetologia. 1985;28(7):412-9. doi: 10.1007/BF00280883. 
17. Chissini RBC, Kuschnir MC, Oliveira CL, Giannini DT, Santos B. Cutoff Values for HOMA-IR Associated With Metabolic Syndrome in the Study of Cardiovascular Risk in Adolescents (ERICA Study). Nutrition. 2020;71:110608. doi: 10.1016/j.nut.2019.110608.

18. Giuliano ICB, Caramelli B, Pellanda L, Duncan B, Mattos S, Fonseca FH, et al. Atualização da Diretriz Brasileira de Dislipidemias e Prevenção de Aterosclerose. Arq Bras Cardiol. 2017;85(Suppl 6):1-76. doi: 10.1590/ S0066-782X2005002500001.

19. Onis M, Onyango AW, Borghi E, Siyam A, Nishida C, Siekmann J. Development of a WHO Growth Reference for School-Aged Children and Adolescents. Bull World Health Organ. 2007;85(9):660-7. doi: 10.2471/ blt.07.043497.

20. Tanner JM. Growth at Adolescence. 2nd ed. Oxford: Blackwell Scientific Publications. 1962.

21. Kaczmarek M, Stawińska-Witoszyńska B, Krzyżaniak A, KrzywińskaWiewiorowska M, Siwińska A. Who is at Higher Risk of Hypertension? Socioeconomic status differences in Blood Pressure Among Polish Adolescents: a Population-Based ADOPOLNOR study. Eur J Pediatr. 2015;174(11):1461-73. doi: 10.1007/s00431-015-2554-0.

22. Yazdanpanah S, Rabiee M, Tahriri M, Abdolrahim M, Rajab A, Jazayeri HE, et al. Evaluation of Glycated Albumin (GA) and GA/HbA1c Ratio for Diagnosis of Diabetes and Glycemic Control: A Comprehensive Review. Crit Rev Clin Lab Sci. 2017;54(4):219-32. doi: 10.1080/10408363.2017.1299684.

23. Saxena T, Ali AO, Saxena M. Pathophysiology of Essential Hypertension: An Update. Expert Rev Cardiovasc Ther. 2018;16(12):879-87. doi: 10.1080/14779072.2018.1540301.

24. Freitas PAC, Ehlert LR, Camargo JL. Glycated Albumin: A Potential Biomarker in Diabetes. Arch Endocrinol Metab. 2017;61(3):296-304. doi: 10.1590/2359-3997000000272.

25. Incalza MA, D'Oria R, Natalicchio A, Perrini S, Laviola L, Giorgino F. Oxidative Stress and Reactive Oxygen Species in Endothelial Dysfunction Associated With Cardiovascular and Metabolic Diseases. Vascul Pharmacol. 2018;100:1-19. doi: 10.1016/j.vph.2017.05.005.

26. Andrade MIS, Oliveira JS, Leal VS, Lima NMDS, Bezerra PB, Santiago ERC, et al. Prevalence of Insulin Resistance and Association With Metabolic Risk Factors and Food Consumption in Adolescents - Recife/Brazil. Rev Paul Pediatr. 2020;38:e2019016. doi: 10.1590/1984-0462/2020/38/2019016.

27. Morais PR, Sousa AL, Jardim TS, Nascente FM, Mendonça KL, Povoa TI, et al. Correlation of Insulin Resistance with Anthropometric Measures and Blood Pressure in Adolescents. Arq Bras Cardiol. 2016;106(4):319-26. doi: 10.5935/abc.20160041.

28. Wang F, Han L, Hu D. Fasting Insulin, Insulin Resistance and Risk of Hypertension in the General Population: A meta-analysis. Clin Chim Acta. 2017;464:57-63. doi: 10.1016/j.cca.2016.11.009.

29. Ghomari-Boukhatem H, Bouchouicha A, Mekki K, Chenni K, Belhadj M, Bouchenak M. Blood Pressure, Dyslipidemia and Inflammatory Factors are Related to Body Mass Index in Scholar Adolescents. Arch Med Sci. 2017;13(1):46-52. doi: 10.5114/aoms.2017.64713.

30. Bozza R, Campos W, Barbosa Filho VC, Stabelini Neto A, Silva MP, Maziero RS. High Blood Pressure in Adolescents of Curitiba: Prevalence and Associated Factors. Arq Bras Cardiol. 2016;106(5):411-8. doi: 10.5935/ abc. 20160044 .
31. Cohen JB. Hypertension in Obesity and the Impact of Weight Loss. Curr Cardiol Rep. 2017 Aug;19(10):98. doi: 10.1007/s11886-017-0912-4.

32. Caillon A, Schiffrin EL. Role of Inflammation and Immunity in Hypertension: Recent Epidemiological, Laboratory, and Clinical Evidence. Curr Hypertens Rep. 2016;18(3):21. doi: 10.1007/s11906-016-0628-7.

33. Seravalle G, Grassi G. Obesity and Hypertension. Pharmacol Res. 2017;122:17. doi: 10.1016/j.phrs.2017.05.013.

34. Xie D, Bollag WB. Obesity, Hypertension and Aldosterone: is Leptin the Link? J Endocrinol. 2016;230(1):7-11. doi: 10.1530/JOE-16-0160.

35. Schütten MT, Houben AJ, Leeuw PW, Stehouwer CD. The Link Between Adipose Tissue Renin-Angiotensin-Aldosterone System Signaling and ObesityAssociated Hypertension. Physiology. 2017;32(3):197-209. doi: 10.1152/ physiol.00037.2016.

36. Weiss TW, Arnesen H, Seljeflot I. Components of the Interleukin-6 Transsignalling System are Associated With the Metabolic Syndrome, Endothelial Dysfunction and Arterial Stiffness. Metabolism. 2013:62(7):100813. doi: 10.1016/j.metabol.2013.01.019. Epub 2013 Feb 19. PMID: 23428306.

37. Alvim RDO, Santos PCJL, Bortolotto LA, Mill JG, Pereira AC. Rigidez Arterial Aspectos Fisiopatológicos e Genéticos. Int J Cardiovasc Sci. 2017; 30(5):43341. 10.5935/2359-4802.20170053.

38. Aiello AM, Mello LM, Nunes MS, Silva AS, Nunes A. Prevalence of Obesity in Children and Adolescents in Brazil: AMeta-analysis of Cross-sectional Studies. Curr Pediatr Rev. 2015;11(1):36-42. doi: 10.2174/1573396311666150501 003250.

39. Song P, Zhang Y, Yu J, Zha M, Zhu Y, Rahimi K, et al. Global Prevalence of Hypertension in Children: A Systematic Review and Meta-analysis. JAMA Pediatr. 2019;173(12):1154-63. doi: 10.1001/jamapediatrics.2019.3310.

40. Matthew B, Flesher M, Sampath S, Nguyen N, Alizadeh-Pasdar N, Barclay K. The effect of intensive preconditioning and close follow-up on bariatric surgery outcomes: Does multidisciplinary care contribute to positive results whether a gastric bypass or sleeve gastrectomy is performed? B C Med J. 2015;57(6):23843.

41. Yang L, Magnussen CG, Yang L, Bovet P, Xi B. Elevated Blood Pressure in Childhood or Adolescence and Cardiovascular Outcomes in Adulthood: A Systematic Review. Hypertension. 2020;75(4):948-55. doi: 10.1161/ HYPERTENSIONAHA.119.14168.

42. Gonçalves VS, Duarte EC, Dutra ES, Barufaldi LA, Carvalho KM. Characteristic of the School food Environment Associated With Hypertension and Obesity in Brazilian Adolescents: A Multilevel Analysis of the Study of Cardiovascular Risks in Adolescents (ERICA). Public Health Nutr. 2019;22(14):2625-34. doi: 10.1017/S1368980019001010.

43. Ronca DB, Blume CA, Cureau FV, Camey SA, Leotti VB, Drehmer M, et al Diet Quality Index for Brazilian Adolescents: The ERICA Study. Eur J Nutr. 2020;59(2):539-56. doi: 10.1007/s00394-019-01923-8.

44. Cureau FV, Silva TL, Bloch KV, Fujimori E, Belfort DR, Carvalho KM, et al ERICA: Leisure-Time Physical Inactivity in Brazilian Adolescents. Rev Saude Publica. 2016;50(Suppl 1):4s. doi: 10.1590/S01518-8787.2016050006683.

45. Lopes CS, Abreu GA, Santos DF, Menezes PR, Carvalho KM, Cunha CF, et al. ERICA: Prevalence of Common Mental Disorders in Brazilian Adolescents. Rev Saude Publica. 2016;50(Suppl 1):14s. doi: 10.1590 S01518-8787.2016050006690. 